\title{
Dark Energy Constraints from a five-dimensional AdS Black Hole via AdS/CFT
}

\author{
Yoobin Jeong ${ }^{2, *}$, Sunly Khimphun ${ }^{1,2, * *}$, Bum-Hoon Lee Le, $^{2, * *}$, and Gansukh Tumurtushaa ${ }^{3, * * * *}$ \\ ${ }^{1}$ Graduate School of Science, RUPP, Cambodia 12150 \\ ${ }^{2}$ Center for Quantum Spacetime (CQUeST), Sogang University, Seoul, Korea 121-742 \\ ${ }^{3}$ Center for Theoretical Physics of the Universe, Institute for Basic Science (IBS), Daejeon 34051, \\ Korea
}

\begin{abstract}
There are many dark energy models having been investigated with constraint from observational data. The best dark energy candidate is $\Lambda \mathrm{CDM}$ model. In this work, our model has extended to include AdS/CFT correspondent, where the AdS black hole boundary exhibit the FLRW metric foliated with four-dimensional gravity theory, can lead to a modified Friedmann Equation contains extra parameter coming from 5D AdS (hairy) black hole, where such extension to $\Lambda \mathrm{CDM}$ can be considered. We obtained modified Friedmann equation from both 5D hairless and scalar hair black hole. The effect from extra dimension in hairless black hole solutions can be observed in the form of radiation. Also, it is interesting to see a non-trivial term emerges in scalar hair solution case. However in this study, we only consider Hairless black hole case for data constraint by using a join analysis of Supernovae, CMB and $H_{0}$ measurement to obtain $\chi_{\min }^{2}$. As a result, we compare this particular case with $\Lambda \mathrm{CDM}$ using AIC and BIC statistics.
\end{abstract}

\section{Boundary of 5 dimensional AdS Black Hole}

We consider the metric

$$
d s^{2}=-f(r) d t^{2}+g(r) d r^{2}+\Sigma(r)^{2} d \Omega_{3}^{2} .
$$

This metric describe $\mathrm{AAdS}_{5}$ when $f(r) \sim \frac{r^{2}}{L^{2}}, g(r) \sim \frac{L^{2}}{r^{2}}$, and $\Sigma \sim \frac{r}{L}$ for large $r$ with the AdS radius $L$. Introducing a new coordinates $v$ such that $d t= \pm \frac{1}{\sqrt{f(r) g(r)}} d v \mp \sqrt{\frac{g(r)}{f(r)}} d r$, we obtain a metric in the Eddington-Finkelstein $(\mathrm{EF})$ coordinates

$$
d s^{2}=2 d v d r-f(r) d v^{2}+\Sigma(r)^{2} d \Omega_{3}^{2},
$$

Adopt a new time and radial coordinates $V$ and $R$ such that $d v=\frac{d V}{a(V)}$ and $R=\frac{r}{a(V)}$ where $a(V)$ will be the scale factor. Then the metric (2) becomes

$$
d s^{2}=2 d V d R-\left[\frac{f(R a)}{a^{2}}-2 R \frac{\dot{a}}{a}\right] d V^{2}+\Sigma(R a)^{2} d \Omega_{3}^{2}
$$

\footnotetext{
*e-mail: physics10@ postech.ac.kr

**e-mail: kpslourk@gmail.com

***e-mail: bhl@sogang.ac.kr

****e-mail: gansuhmgl@ibs.re.kr
} 
where this metric behave as FLRW metric if $f(R a)$ and $\Sigma(R a)$ behave as $\mathrm{AAdS}_{5}$. Introduce Fefferman-Graham(FG) coordinates (This coordinate can have well-defined stress-energy tensor in dual CFT)

$$
d s^{2}=\frac{L^{2}}{z^{2}}\left[d z^{2}+g_{\mu \nu} d x^{\mu} d x^{v}\right]
$$

with

$$
g_{\mu \nu}(z, x)=g_{\mu \nu}^{(0)}(x)+z^{2} g_{\mu \nu}^{(2)}(x)+z^{4}\left(g_{\mu \nu}^{(4)}(x)+h_{\mu \nu}^{(4)}(x) \log z\right)+\cdots,
$$

Comparing (EF) and (FG) coordinate, we should find the relation for metric component in FG coordinate in terms of black hole information from EF coordinate by expansion near boundary. EF will ensure that we will have FLRW metric behavior near the boundary of Black Hole, while FG will ensure that we may have a well-defined dual field theory for 4D gravity. Such relation is given as

$$
\begin{aligned}
& 2 \partial_{z} R \partial_{z} V-\alpha\left(\partial_{z} V\right)^{2}=\frac{L^{2}}{z^{2}}, \\
& \partial_{z} V \partial_{\tau} R+\partial_{\tau} V \partial_{z} R-\alpha \partial_{z} V \partial_{\tau} V=0, \text { where } \alpha=\left[\frac{f(R a)}{a^{2}}-2 R \frac{\dot{a}}{a}\right] \\
& g_{\tau \tau}=-\frac{\left(\partial_{\tau} V\right)^{2}}{\left(\partial_{z} V\right)^{2}}, \quad g_{i j} d x^{i} d x^{j}=\frac{z^{2}}{L^{2}} \Sigma^{2}(R a) d \Omega_{3}^{2} .
\end{aligned}
$$

Our action includes black hole bulk gravity in 5D whose boundary is a usual 4D gravity with FLRW background metric.

$S=\int_{\mathcal{M}} d^{5} x \sqrt{-\operatorname{det} g_{5}} \mathcal{L}_{5 \mathrm{D}}^{\text {gravity }}+\frac{1}{16 \pi G_{4}} \int_{\partial \mathcal{M}} d^{4} x \sqrt{-\operatorname{det} g^{(0)}}\left(R-2 \Lambda_{4}\right)+\int_{\partial \mathcal{M}} d^{4} x \sqrt{-\operatorname{det} g^{(0)}} \mathcal{L}_{4 \mathrm{D}}^{\text {matter }}$.

As a result, equation of motion associated with mixed boundary condition should be expressed as [1]

$$
R_{\mu \nu}-\frac{1}{2} g_{\mu \nu}^{(0)} R+\Lambda_{4} g_{\mu \nu}^{(0)}=8 \pi G_{4}\left(\left\langle T_{\mu \nu}^{\mathrm{CFT}}\right\rangle+T_{\mu \nu}^{\mathrm{matter}}\right)
$$

Notice that $\left\langle T_{\mu \nu}^{\mathrm{CFT}}\right\rangle$ can be obtained from AdS/CFT correspondent. Above method can be found in $[2,3]$

\section{Example of Hariy Black Hole Case}

Consider the action [4]

$$
S=\frac{1}{2 \kappa^{2}} \int d^{5} x \sqrt{-g}\left[R-W(\phi) F^{2}-\frac{1}{2}(\partial \phi)^{2}-V(\phi)\right]
$$

with a potential $V(\phi)$ and a coupling $W(\phi)$ of the form

$$
V(\phi)=-\frac{1}{L^{2}}\left(8 e^{\phi / \sqrt{6}}+4 e^{-2 \phi / \sqrt{6}}\right), \quad W(\phi)=\frac{1}{4} e^{2 \phi / \sqrt{6}},
$$

and metric

$$
d s^{2}=e^{2 C}\left(-h d t^{2}+d \vec{x}^{2}\right)+\frac{e^{2 D}}{h} d r^{2}
$$


where

$$
\begin{aligned}
& C=\log \left(\frac{r}{L}\right)+\frac{1}{3} \log \left(1+\frac{Q^{2}}{r^{2}}\right), \quad D=-\log \left(\frac{r}{L}\right)-\frac{2}{3} \log \left(1+\frac{Q^{2}}{r^{2}}\right) \\
& h=1-\frac{M L^{2}}{\left(Q^{2}+r^{2}\right)^{2}}, \quad \phi=\frac{2}{\sqrt{6}} \log \left(1+\frac{Q^{2}}{r^{2}}\right), \quad A=\left(-\frac{Q \sqrt{2 M}}{Q^{2}+r^{2}}+\frac{Q \sqrt{2 M}}{Q^{2}+r_{h}^{2}}\right) d t .
\end{aligned}
$$

Here $Q$ is the charge and $M$ is the mass of the black hole. $r_{h}$ is the horizon, which is defined by $h\left(r_{h}\right)=0$. The well-defined stress-energy tensor for this black hole solution is given by [5]

$$
\begin{aligned}
\left\langle T_{\mu \nu}^{\mathrm{CFT}}\right\rangle= & \frac{2}{\kappa^{2}}\left(g_{(4) \mu \nu}-\operatorname{Tr} g_{(4)} g_{(0) \mu \nu}\right)+\frac{1}{\kappa^{2}}\left(h_{(4) \mu \nu}-\frac{1}{2} \operatorname{Tr} h_{(4)} g_{(0) \mu \nu}\right)-\frac{1}{\kappa^{2}}\left(\operatorname{Tr} g_{(2)} g_{(2) \mu \nu}-\frac{1}{2} \operatorname{Tr}\left(g_{(2)}^{2}\right) g_{(0) \mu \nu}\right) \\
+ & \frac{1}{2 \kappa^{2}}\left(D_{(0) \sigma} D_{(0)(\mu} g_{(2) \nu)}-\frac{1}{2} \square_{(0)} g_{(2) \mu \nu}-\frac{1}{2} D_{(0) \mu} D_{(0) \nu} \operatorname{Tr} g_{(2)}-\partial_{(\mu} \phi_{(0)} \partial_{v)} \phi_{(2)}\right. \\
& -\frac{1}{2} g_{(2) \mu \nu}\left(R\left[g_{(0)}\right]-\frac{1}{2} \partial_{\sigma} \phi_{(0)} \partial^{\sigma} \phi_{(0)}\right)+\frac{1}{2} g_{(0) \mu \nu} g_{(2)}^{\sigma \rho}\left(R\left[g_{(0)}\right]_{\sigma \rho}-\frac{1}{2} \partial_{\sigma} \phi_{(0)} \partial_{\rho} \phi_{(0)}\right) \\
& \left.-\frac{1}{2} g_{(0) \mu \nu}\left(D_{(0) \sigma} D_{(0) \rho} g_{(2)}^{\sigma \rho}-\square_{(0)} \operatorname{Tr} g_{(2)}-\partial_{\sigma} \phi_{(0)} \partial^{\sigma} \phi_{(2)}\right)\right)+\frac{1}{48} F_{\sigma \rho}^{(0)} F_{(0)}^{\sigma \rho} g_{\mu \nu}^{(0)} .
\end{aligned}
$$

With the above background solutions, the explicit formula for the stress-energy tensor is

$$
\begin{aligned}
\left\langle T_{\tau \tau}^{\mathrm{CFT}}\right\rangle & =\frac{24 Q^{2} \dot{a}^{2}+9 \dot{a}^{4}+8 Q^{4}+36 M}{192 \pi \mathrm{G}_{5} a^{4}} \\
\left\langle T_{i j}^{\mathrm{CFT}}\right\rangle & =\frac{-48 Q^{2} a \ddot{a}+9 \dot{a}^{4}+12 \dot{a}^{2}\left(2 Q^{2}-3 a \ddot{a}\right)+8 Q^{4}+36 M}{576 \pi \mathrm{G}_{5} a^{2}} \delta_{i j} .
\end{aligned}
$$

The $\tau \tau$-component of the Einstein equation (8) gives a modified Friedmann equation

$$
H^{2}=\frac{\beta}{8}\left(H^{4}+\frac{8 Q^{2}}{3 a^{2}} H^{2}+\frac{36 M+8 Q^{4}}{9 a^{4}}\right)+\frac{8 \pi G_{4}}{3} \rho+\frac{\Lambda_{4}}{3} .
$$

Here, mass $M$ and $Q$ contribute to radiation term. The $Q^{2}$ in $H^{2} / a^{2}$ term in (15) does not appear in RN-AdS black hole [6], which could be more interesting than the other black holes during late time. Notice that above equations will recover $\Lambda \mathrm{CDM}$ when $\beta \equiv \frac{G_{4}}{G_{5}} \rightarrow 0$

\section{Observational Constraint}

Rewrite (15) and solve for $H^{2}$, we have

$$
H^{2}=\frac{1}{2 \Omega_{\beta}}\left[\frac{1}{H_{0}^{2}}-\Omega_{Q} a^{-2} \pm\left(\left(\Omega_{Q} a^{-2}-\frac{1}{H_{0}^{2}}\right)^{2}-4 \Omega_{\beta}\left(\left(\Omega_{M}+\Omega_{r}\right) a^{-4}+\Omega_{m} a^{-2}+\Omega_{\Lambda}\right)\right)\right],
$$

where

$$
\Omega_{\beta}=\frac{\beta}{8 H_{0}^{2}}, \quad \Omega_{Q}=\frac{8 Q^{2}}{3} \Omega_{\beta}, \quad \Omega_{M}=\frac{36 M+8 Q^{4}}{9} \Omega_{\beta}, \quad \Omega_{m, r}=\frac{8 \pi G_{4} \rho_{m, r}^{0}}{3 H_{0}^{2}}, \quad \Omega_{\Lambda}=\frac{\Lambda_{4}}{3 H_{0}^{2}}
$$

The expression in (16) is all we need to check our model with observational constraint. 


\section{Supernovae, CMB and $H_{0}$ measurement Data}

We compute $\chi_{\min , \mathrm{SN}}^{2}$ following the JLA compilation [7] which has been shown to be statistically stable [8]. We compute $\chi_{\text {min,CMB }}^{2}$ using shift parameter obtained in [9] which base on the Planck 2015 [10]. We also constraint our model using $H_{0}$ data derived in [11]. To study different dark energy models, $H_{0}$ measurement should be considered, as motivated in [12].

\section{AIC and BIC}

We have

$$
\mathrm{AIC}=-2 \ln \mathcal{L}_{\text {max }}+2 k, \mathrm{BIC}=-2 \ln \mathcal{L}_{\max }+k \ln N
$$

where $\mathcal{L}_{\text {max }}$ is the maximum likelyhood $\left(\chi_{\text {min }}^{2}=-2 \ln \mathcal{L}_{\text {max }}\right), k$ is the number of parameters, and $N$ is the number of data points used in the fit. We compare AIC and BIC of our model with $\Lambda \mathrm{CDM}$ as a reference model with $\chi_{\min }^{2}=695.365$

\begin{tabular}{|l||l|l|l|l|}
\hline$\Lambda$ CDM as a reference & $\Delta$ AIC & $\Delta$ BIC & $\chi_{\min }^{2}$ & Extra Parameter \\
\hline Hairless Black Hole & -0.028 & 4.5827 & 693.337 & 1 \\
\hline
\end{tabular}

\section{Conclusions}

We have obtained modified Friedmann equations by implementing mixed boundary condition and adopting AdS/CFT correspondent. New terms associated with the charge $Q$ emerge in this scalar hair solution which has not been observed in RN-AdS black hole. In the limit where $\beta H^{4} \ll 1$, such analysis can be done easily to see that for one of the solutions in (16), scale factor $a(t)$ has the same characteristic as $\Lambda$ CDM. After that, we constraint our model using Supernovae, $\mathrm{CMB}$ and $H_{0}$ data and compare with $\Lambda \mathrm{CDM}$ using information criterion (AIC and BIC). Schwarzschild black hole case shows that we obtained smaller $\chi_{\min }^{2}$ and AIC than $\Lambda$ CDM. It is usually the case when a model has more parameter, it can lead to smaller $\chi_{\min }^{2}$ which is the reason why information criterion is needed to penalize the extra parameter. $\triangle \mathrm{AIC}$ we obtained shows that our model is not ruled out by current data, but instead is competitive with $\Lambda \mathrm{CDM}$ from the perspective of data fitting. As a result, it also has the best data fitting comparing to the other dark energy models. $\triangle \mathrm{BIC}$, however, shows that $\Lambda C D M$ is still a model which has a tendency to be close to the true model slightly better than this current model even though our model tends to have a slightly better data fitting. More test can be done after more reliable and stable data are available to further compare and understand.

\section{References}

[1] G. Compere and D. Marolf, Class. Quant. Grav. 25, 195014 (2008)

[2] G. Camilo, Eur. Phys. J. C 76, no. 12, 682 (2016)

[3] P. S. Apostolopoulos, G. Siopsis and N. Tetradis, Phys. Rev. Lett. 102, 151301 (2009)

[4] B. S. Kim, JHEP 1611, 044 (2016)

[5] I. Papadimitriou, JHEP 1108, 119 (2011)

[6] S. Banerjee, S. Bhowmick, A. Sahay and G. Siopsis, Class. Quant. Grav. 30, 075022 (2013)

[7] M. Betoule et al. [SDSS Collaboration], Astron. Astrophys. 568, A22 (2014) 
[8] U. Alam and J. Lasue, JCAP 1706, no. 06, 034 (2017)

[9] Y. Wang and M. Dai, Phys. Rev. D 94, no. 8, 083521 (2016)

[10] P. A. R. Ade et al. [Planck Collaboration], Astron. Astrophys. 594, A13 (2016)

[11] A. G. Riess et al., Astrophys. J. 730, 119 (2011) Erratum: [Astrophys. J. 732, 129 (2011)]

[12] Y. Y. Xu and X. Zhang, Eur. Phys. J. C 76, no. 11, 588 (2016) 